\title{
Begleiteter Suizid zur Prime Time: Das „Recht zu Sterben“
}

\author{
Assisted Suicide in Prime Time: The „Right to Die“
}

Autoren

Institute
Tanja Neuner $^{1}$, Bettina Hübner-Liebermann ${ }^{1}$, Hermann Spießl ${ }^{1,2}{ }^{2}$, Helmut Hausner ${ }^{1}$

Klinik und Poliklinik für Psychiatrie, Psychosomatik und Psychotherapie der Universität am Bezirksklinikum Regensburg

Klinik für Psychiatrie, Psychotherapie und Psychosomatik, Bezirkskrankenhaus Landshut
Bibliografie

DOI $10.1055 / \mathrm{s}-0028-1090151$

Psychiat Prax 2009; 36:

155-156

(c) Georg Thieme Verlag KG

Stuttgart · New York .

ISSN 0303-4259

Korrespondenzadresse Dipl.-Psych. Tanja Neuner Klinik und Poliklinik für Psychiatrie, Psychosomatik und Psychotherapie der Universität am Bezirksklinikum Regensburg 93042 Regensburg tanja.neuner@medbo.de
„I love you, sweetheart, so much.“ - mit diesen Worten verabschiedete sich der 59-jährige USBürger Craig Ewert in der am 10. Dezember bei Sky Real Lives in Großbritannien ausgestrahlten Fernsehdokumentation „Recht zu Sterben“ von seiner Frau. Die Dokumentation zeigt den begleiteten Suizid des an amyotropher Lateralsklerose erkrankten pensionierten Universitätsprofessors in einer Klinik der Schweizer Sterbehilfeorganisation „Dignitas“. Zu guter Letzt nimmt Ewert zur besten Sendezeit um 21 Uhr einen Becher mit Barbituraten zu sich und betätigt mit dem Mund eine Zeitschaltuhr, die 45 Minuten später sein Beatmungsgerät abschaltet.

\section{Begleiteter Suizid im Fernsehen: Aufklärung kontra Suizidpråvention? $\nabla$}

Während Sky Real Lives die Dokumentation als „lehrreichen Einblick“ bezeichnet, ist die Ausstrahlung eines begleiteten Suizids einer erfolgreichen Suizidprävention wenig zuträglich. Nachahmungstaten aufgrund des Werther-Effektes - einem Anstieg von Suiziden nach Veröffentlichung detaillierter Berichterstattungen in den Medien - sind seit Langem wissenschaftlich belegt. Nach Ausstrahlung der Fernsehsendung „Tod eines Schülers“ im Jahr 1981 stieg beispielsweise die Zahl der Bahnsuizide in Deutschland um bis zu $175 \%$, am deutlichsten in der altersund geschlechtskongruenten Gruppe der 15-19-jährigen männlichen Schüler. Auch bei erneuter Ausstrahlung der sechsteiligen Serie eineinhalb Jahre später zeigte sich ein Werther-Effekt mit einem Anstieg der Suizide Jugendlicher um 115\% [1]. Ähnliche Ergebnisse fanden sich für U-Bahn-Suizide in Österreich [2]. Nachdem in Wien Mitte der 80er-Jahre in den Medien gehäuft über U-Bahn-Suizide berichtet wurde, vereinbarte die Österreichische Gesellschaft für Suizidprävention (ÖGS) mit den Medien, von einer
Berichterstattung über Suizide abzusehen. Daraufhin reduzierte sich ab 1987 die Zahl der U-Bahn-Suizide um über $70 \%$.

Aufgrund dieser Erfahrungen ist anzunehmen, dass sich auch für begleitete Suizide im Rahmen der Sterbehilfe ein ähnlicher Effekt zeigen könnte. Erste Belege hierfür kommen aus der Schweiz [3]: Nach der ausführlichen Berichterstattung über den begleiteten Suizid eines prominenten Paares in den Medien konnte in Basel ein beträchtlicher Anstieg der mittels der Sterbehilfeorganisation EXIT ausgeführten begleiteten Suizide - hauptsächlich in der Gruppe der Frauen über 65 Jahre - verzeichnet werden. Es kam zu signifikant mehr begleiteten Suiziden in den zwei Jahren nach dem kritischen Ereignis als in den zwei Jahren davor. Vor allem die beinahe schon enthusiastische Berichterstattung könnte diesen Werther-Effekt induziert haben. Auch im Fall Craig Ewert liegt aus unserer Sicht eine erhebliche Gefahr in der friedlich anmutenden, mit Beethovenklängen unterlegten „ästhetischen“ Darstellung des Suizids: Man muss davon ausgehen, dass weitere unheilbar erkrankte Menschen zu dem Schluss verleitet werden, auch für sie wäre der Suizid eine angemessene Problemlösung. Ein Werther-Effekt nach Ausstrahlung von „Recht zu Sterben“ kann von daher nicht ausgeschlossen, sondern muss vielmehr befürchtet, werden.

Die Deutsche Gesellschaft für Suizidprävention (DGS) weist in ihren Empfehlungen für die Berichterstattung in den Medien [4] darauf hin, dass alles unterlassen werden sollte, was zur Identifikation mit dem Suizidenten führen kann, z.B. den Suizid als nachvollziehbare, unausweichliche Reaktion oder gar positiv darzustellen. Ebenso kritisch ist eine romantisierende oder idealisierende Darstellung des Suizids zu werten. Die Worte von Barbara Gibbons, SenderChefin von Sky Real Lives, dürften kaum zu einer Ent-Idealisierung beigetragen haben: „By any standards, the decision to take your own life ta- 
kes a great deal of courage. To share this moment with a TV audience, as Craig Ewert did, and manage to remain articulate right up until the end, takes exceptional courage.“

\section{Patienten im Terminalstadium als Ausnahme? $\nabla$}

Über $90 \%$ der Suizide beruhen auf einer psychischen Erkrankung [5], wobei die Depression mit 40-70\% aller Suizide an der Spitze liegt [6].

Zwar sind die Suizidimpulse körperlich schwer kranker Menschen nicht ohne Weiteres als eine psychiatrische Erkrankung zu interpretieren, jedoch weisen aktuelle Studien auch für diese Patientengruppe einen engen Zusammenhang zwischen dem Wunsch nach Sterbehilfe und dem Vorliegen psychiatrischer Störungen, insbesondere einer Depression, nach. In einer Stichprobe von AIDS-Patienten im fortgeschrittenen Krankheitsstadium prädizierten Hoffnungslosigkeit und Depression den Wunsch zu sterben [7]. Auch bei Krebspatienten mit infauster Prognose ist das Verlangen nach ärztlich begleitetem Suizid mit der Diagnose einer „Major Depression“ assoziiert [8]. Dies gilt auch für Patienten mit amyotropher Lateralsklerose: Die Bereitschaft zu begleitetem Suizid bei ALS geht mit einer deutlichen depressiven Symptomatik und Hoffnungslosigkeit einher [9]. Diese Ergebnisse heben eindrucksvoll hervor, dass auch bei Patienten im Terminalstadium einer primär somatischen Erkrankung der psychische Status sorgfältig exploriert werden muss. Andernfalls ist ein ausreichender Schutz vor Fehlentscheidungen der Patienten nicht möglich, da die Freiheit ihrer Wahl durch eine depressive Erkrankung beeinflusst ist. Ob Sterbehilfeorganisationen daher die Freiheit des Kranken eher schützen oder letztlich doch einschränken, bleibt vor diesem Hintergrund fraglich.

Ist die soziale Hemmschwelle „Suiziddokumentationen“ im Fernsehen auszustrahlen, erst einmal überschritten, könnte die weitere Verbreitung solcher Einschaltquoten bringender Berichte ungünstige Folgen für die kollektive Wahrnehmung des Themas haben: die regelmäßige Ausstrahlung von „Suiziddokumentationen" induziert die Wahrnehmung eines Suizids als alltägliche Normalität - das Gefühl, dass ein begleiteter Suizid seinen festen Platz in der Gesellschaft habe, nichts Außergewöhnliches und ein jederzeit einzuforderndes Recht sei. Andererseits könnten durch das öffentliche Propagieren einer aktiven Sterbehilfe und den damit einhergehenden Folgen auch gerade die Schwächeren und Älteren weiter an den Rand der Gesellschaft gedrängt und unter Entscheidungsdruck gesetzt werden. Vielleicht kommen auch sie - ähnlich wie Craig Ewert - zu dem „logischen Schluss“, niemandem mehr zur Last fallen zu wollen: „If I do not go through with it then my choice is to suffer and to enforce suffering on my family [...].“

\section{Rechtslage zur assistierten oder aktiven Sterbehilfe \\ $\nabla$}

International ist die Rechtslage zur aktiven oder assistierten Sterbehilfe uneinheitlich. In den Niederlanden beispielsweise ist die Sterbehilfe explizit gesetzlich geregelt. In Situationen aussichts- und ausweglosen Leidens bleibt der Sterbehelfer straffrei, wenn ein einwilligungsfähiger Patient die aktive Sterbehilfe einfordert und vorher ein zweiter unabhängiger Arzt das Vorliegen der medizinischen Voraussetzungen bestätigt hat. Selbst Min- derjährigen steht diese Entscheidung ab dem 16. Lebensjahr offen, mit Zustimmung der Eltern sogar Kindern ab dem 12. Lebensjahr [10]. Auch die Rechtsprechung in Japan toleriert in vergleichbaren Fällen die Durchführung aktiver Sterbehilfe, allerdings ohne gesetzliche Regelung [11]. Diese Regelungen stellen aber seltene Ausnahmen im Konzert der unterschiedlichen nationalen Rechtsordnungen dar, in den meisten Staaten ist die aktive Tötung von Patienten bis heute jedoch unzulässig.

Nach deutschem Recht ist zwischen assistierter Sterbehilfe im Sinne einer bloßen Beihilfe zur Selbsttötung und aktiver Sterbehilfe zu unterscheiden [12]. Das Bereitstellen eines Tötungsmittels auf Wunsch des Patienten bleibt grundsätzlich straffrei, solange der Betroffene den gesamten Geschehensablauf kontrolliert und beherrscht - also die sogenannte „Tatherrschaft“ im Sinne des deutschen Strafrechts inne hat. Davon ist die echte aktive Sterbehilfe abzugrenzen: Eine unmittelbare Tötungshandlung auch auf ausdrücklichen Wunsch des Patienten wird nach §216 StGB als „Tötung auf Verlangen“ mit einer Freiheitsstrafe von sechs Monaten bis zu fünf Jahren geahndet. Bereits der Versuch einer solchen Handlung wird bestraft.

Eine separate Betrachtung ist bei psychisch erkrankten Patienten mit Suizidwunsch [13] erforderlich. Hier ist der Wunsch nach Beihilfe zur Selbsttötung regelmäßig nicht als freiverantwortliche Entscheidung, sondern als typisches Symptom der Erkrankung - beispielsweise einer schweren depressiven Episode oder einer Schizophrenie - zu werten. Käme in diesem Kontext ein behandelnder Arzt dem Ansinnen des Patienten nach, so läge hierin keine straflose Beihilfehandlung mehr, sondern eine strafbare Tötung durch Unterlassung in Garantenstellung nach $\S 13$ StGB. Der Arzt hat nämlich gegenüber dem psychisch Erkrankten die Rechtspflicht dafür einzustehen, dass sich die speziellen Risiken der Erkrankung (z. B. die Suizidalität) nicht realisieren. Wenn dann aktiv unterstützt wird, was eigentlich verhindert werden muss, wird die Grenze zur Strafbarkeit überschritten.

\section{Literatur}

1 Schmidtke A, Häfner $H$. The Werther effect after television films: new evidence for an old hypothesis. Psycho Med 1998; 18: 665-676

2 Sonneck G, Etzersdorfer E, Nagel-Kuess S. Imitative suicide on the Viennese subway. Soc Sci Med 1994; 38: 453-457

3 Frei A, Schenker T, Finzen A et al. The Werther effect and assisted suicide. Suicide Life Threat Behav 2003; 33: 192-200

$4 \mathrm{http}: / /$ www.suizidprophylaxe.de/Medienempfehlung\%20DGS.pdf

5 Moscicki EK. Identification of suicide risk factors using epidemiologic studies. Psychiatr Clin North Am 1997; 20: 499-517

6 http://www.buendnis-depression.de/depression/suizidalitaet.php

7 Rosenfeld B, Breitbart W, Gibson C et al. Desire for hastened death among patients with advanced AIDS. Psychosomatics 2006; 47: 504-512

8 Wilson KG, Chochinov HM, McPherson CJ et al. Desire for euthanasia or physician-assisted suicide in palliative cancer care. Health Psychol 2007; 26: 314-323

9 Achille MA, Ogloff JR. Attitudes toward and desire for assisted suicide among persons with amyotrophic lateral sclerosis. Omega 2003-2004; 48: 1-21

10 Finger C. Evaluation der Praxis der aktiven Sterbehilfe und der Hilfe bei der Selbsttötung in den Niederlanden für das Jahr 2001. MedR 2004; 22: 379-382

11 Deutsch E, Spieckhoff A. Medizinrecht. Springer, 2008: 420

12 Schmidt J, Giring J. Arztstrafrecht. In: Ratzel R, Luxenburger B, Hrsg. Handbuch Medizinrecht. DeutscherAnwaltVerlag, 2008: 814

13 Purucker M, Franke C, Stich J et al. Zweimaliger Suicidversuch eines schizophren Erkrankten während stationärer Therapie. Psychiat Prax 2007; 34 (Suppl. 1): S82-S83 